Original Research

\title{
Fabrication and Demonstration of a 3D-printing/PDMS Integrated Microfluidic Device
}

Jeonghyeon Cheon ${ }^{\ddagger}$, Seunghyun Kim *

Department of Electrical and Computer Engineering, Baylor University, Waco, TX, USA; E-Mails: mpcjh90@gmail.com; Seunghyun_kim@baylor.edu

$\ddagger$ Current Affiliation: POSCO, Pohang, South Korea

* Correspondence: Seunghyun Kim; E-Mail: Seunghyun kim@baylor.edu

Academic Editor: Yifei Jin

Special Issue: 3D Printing of Engineering Materials

Recent Progress in Materials

2022, volume 4, issue 1

doi:10.21926/rpm.2201002
Received: October 21, 2021

Accepted: January 20, 2022

Published: January 25, 2022

\begin{abstract}
3D printing is an attractive method to fabricate microfluidic devices due to (1) its fast and simple process without specialized equipment and cleanroom environment, and (2) its capability to create complex 3D structures. Combined with Polydimethylsiloxane (PDMS), it can be used to develop various microfluidic devices taking advantage of both 3D printing and PDMS. In this paper, we investigated a Digital Light Processing (DLP) 3D printer to fabricate 3D printing/PDMS integrated microfluidic devices. We used it to fabricate both a master mold for the PDMS process and a substrate containing pneumatic ports and channels. The optimal design parameters to print a symmetrical microchannel structure were determined. We also measured the printing accuracy of taper structures as an example of its capability to fabricate complex structures. Then, we fabricated a microfluidic device by integrating a PDMS component with a 3D printed substrate. The microfluidic device operation was demonstrated using dye solutions. The fluidic control results clearly show the microfluidic device works as expected.
\end{abstract}

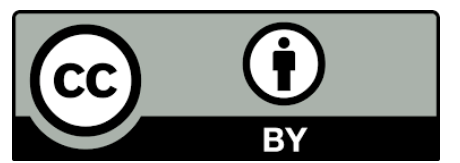

(C) 2022 by the author. This is an open access article distributed under the conditions of the Creative Commons by Attribution License, which permits unrestricted use, distribution, and reproduction in any medium or format, provided the original work is correctly cited. 


\section{Keywords}

DLP 3D printer; PDMS; 3D structure; microfluidic device

\section{Introduction}

Microfluidic devices are miniaturized fluid and gas control systems developed for various applications in chemistry and biology such as lab on a chip (LOC) or micro total analysis systems ( $\mu$ TAS) [1, 2], genetic analysis [3], cell analysis [4], organs on chips [5], drug discovery [6], and biosensing $[7,8]$. Polydimethylsiloxane (PDMS) is the most commonly used material for microfluidic devices [9-17]. PDMS-based microfluidic devices are typically fabricated by bonding a soft lithography-processed PDMS piece on a substrate such as glass, silicon, or PDMS. For soft lithography, a proper mixture of PDMS base and curing agent is poured on top of a mold master, cured, and peeled off from the mold to replicate the structure on PDMS. A mold master is typically fabricated using a photolithography process [18], which transfers patterns on a photomask to a photoresist through UV light exposure. Often, the photolithography process is also used to create structures on the microfluidic device substrate that is bonded with the PDMS. Therefore, the photolithography process is key to fabricating PDMS-based microfluidic devices. While the photolithography process is simple and straightforward, it has some limitations: (1) it requires multiple steps using various chemicals and specialized equipment in a cleanroom environment, and (2) it mainly produces a single step structure making it hard to create complex 3D structures in the vertical direction.

Recently, 3D printing has come to focus as a new method to produce microfluidic devices [1925]. With 3D printing, microfluidic chips can be fabricated in a single step at a low cost without photolithography processes in a cleanroom environment [22-25]. Fused Deposition Modeling (FDM), Stereolithography Apparatus (SLA), and Digital Light Processing (DLP) 3D printers have been widely used for microfluidic device fabrications. Combining 3D printed structures with conventional PDMS structures makes it possible to develop useful microfluidic devices that take advantage of both. For example, Gross et al. demonstrated the design and fabrication of a 3D-printed microfluidic device coated with PDMS for electrical cell lysis [26]. They used PDMS to immobilize a cell layer on it because of its known surface properties instead of an unknown 3D-printed surface. Brennan et al. fabricated an SLA 3D-printed device combined with gas permeable PDMS membranes to control oxygen levels for cell cultures [27]. Tsuda et al. developed a flow selector device using an FDMbased 3D printer and PDMS [28]. They took advantage of both by fabricating the overall device with a 3D printer while a flexible PDMS membrane valve was used for the fluid control.

DLP 3D printer has a great potential to fabricate master molds for the PDMS process and substrates with 3D structures for microfluidic devices due to its fast printing speed, high resolution, capability to create complex 3D structures, and smooth printed surface. Previously, we demonstrated the bonding process between a PDMS piece fabricated with a DLP-printed master mold and a DLP-printed substrate using a thin $\mathrm{SiO} 2$ intermediate layer with the bonding strength $>436.65 \mathrm{kPa}$ [29].

In this paper, we present (1) the optimal design parameters of a DLP 3D printer to create a symmetrical microchannel structure to be used as PDMS master molds, (2) the printing accuracy of 
a DLP 3D printer for taper structures as an example of its capability to create complex 3D structures in the vertical direction, and finally (3) the demonstration of a 3D printing/PDMS integrated microfluidic device for which a DLP 3D printer was used to fabricate the PDMS master mold and substrate.

\section{Materials and Methods}

\subsection{D Printer and the Calibration Process}

B9 Creator (B9Creations LLC, Rapid City, SD) is the DLP 3D printer used for our work. It has an XY resolution of 30,50 , or $70 \mu \mathrm{m}$, and its vertical resolution depends on the resin type. We performed a three-step calibration process before printing a device. In the first calibration step, the build table was calibrated to ensure that it makes good contact with Polydimethylsiloxane (PDMS) layer inside the VAT. Then, the projector was calibrated to set the zoom, focus, and position for the XY resolution used. We used $30 \mu \mathrm{m}$ for the $\mathrm{XY}$ resolution for the calibration process. Finally, a calibration structure was printed. For the $X Y$ resolution of $30 \mu \mathrm{m}$, the calibration structure has a dimension of $50 \mathrm{~mm}$ ( $X$ direction) $\times 30 \mathrm{~mm}$ ( $Y$ direction) $\times 3.5 \mathrm{~mm}$ ( $Z$ direction). We measured the $X Y$ dimensions of the square posts at four corners and the center of the calibration structure (total five square posts). Based on the measurements, we adjusted the balance (for the left and right sides), the fade (for the top and bottom sides), and the Machine Specific Multiplier (for overall exposure time) to obtain the $X Y$ dimensions of the five square posts within the tolerance $( \pm 30 \mu \mathrm{m})$ of the design $(5 \mathrm{~mm} \times 5 \mathrm{~mm})$.

\subsection{Materials}

Various resins are available through the B9 Creator manufacturer, while third-party resins can also be used. Out of many resins, We chose the manufacturer's black resin to print the mold master because it has less thermal deformation compared to other resins. This property is important for the mold master print since it has to go through the PDMS heat curing process to create the PDMS part of the microfluidic device. The black resin has a minimum $Z$ resolution of $30 \mu \mathrm{m}$. For printing the substrate part of the microfluidic device, we chose the manufacturer's yellow resin due to its small vertical resolution $(20 \mu \mathrm{m})$ compared to other resins. SYLGARD 184 Silicone Elastomer Kit (DOW, MI) is used for fabricating the PDMS part. Trimethylchlorosilane (TMCS) (SIGMA-ALDRICH, $\mathrm{MO}$ ) is used to prevent the PDMS from sticking to the mold master.

\subsection{D Printer Settings}

For both the mold master and substrate, an $X Y$ resolution of $30 \mu \mathrm{m}$ was used. The $Z$ resolution of the black resin was $30 \mu \mathrm{m}$ to print the mold master, while $20 \mu \mathrm{m}$ was used for the $Z$ resolution of the yellow resin to print the substrate. The light intensity of the projector was not adjustable. We used the manufacturer recommended exposure time for printing. For the mold master printing with the black resin, the exposure time was 0.432 seconds, while the exposure time for the substrate printing with the yellow resin was 1.366 seconds. 


\section{Results and Discussions}

\subsection{Design Optimization for DLP 3D Printer to Create a Symmetrical Microchannel with Target Dimensions}

PDMS-based microfluidic devices often include membrane type valves to control the fluid flow in the microchannel. For the proper operation of valves, it is necessary to create a rounded and symmetric microchannel so that the channel can be completely closed by a thin PDMS membrane when the valve is actuated. As a first step to fabricating a PDMS mold master with B9 Creator, we performed experiments to determine the optimal print parameters of B9 Creator to print a rounded and symmetric microchannel with a channel dimension of $200 \mu \mathrm{m}$ as shown in Figure 1(a) (solid line).

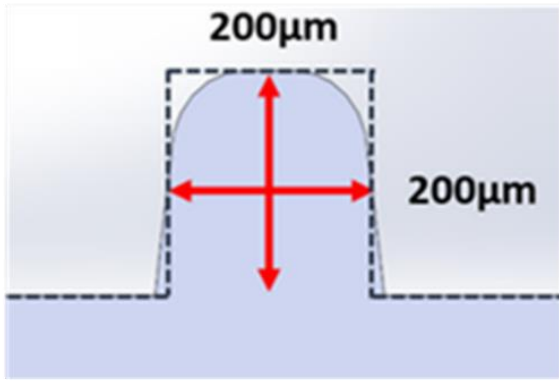

a

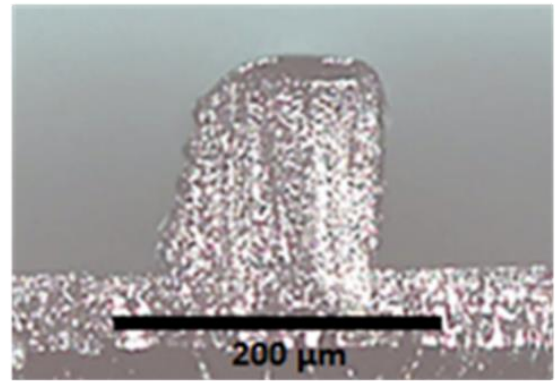

b

Figure 1 (a) Schematic of the Solidworks design (dashed line) with the target shape and (b) cross sectional view of a printed structure.

Even though we performed the calibration process before printing, the discrepancy between the design and the print of 3D printing is anticipated within the tolerance. Therefore, fine-tuning in the scale of microfluidic channel dimensions is necessary to achieve the desired dimensions. The rounding effect is also commonly found from our own testing prints and others using DLP 3D printers which may be due to the variations in exposure energy [30]. With the anticipated rounding effect and discrepancy, we tested a rectangular design first with a height of $240 \mu \mathrm{m}$ and a height of $120 \mu \mathrm{m}$ as shown in Figure 1 (a) (dashed line). Solidworks (Dassault Systems, France) is used for the design. Before printing, the resin was shaken for 6 minutes on an orbital shaker at $160 \mathrm{rpm}$ and then heated in a water bath. The heated resin was then poured into the leveled DLP 3D printer VAT. After printing, the printed structure was rinsed with DI water and Isopropyl alcohol (IPA). Then, the printed structure was dried with nitrogen followed by curing for 30 seconds in the resin curing box. We then inspected the printed structure using a microscope. A microscope image showing the crosssectional view of the printed structure with a rectangular shape design is shown in Figure 1 (b). The left side of the channel was rounded while the right side of it was not, making the channel structure asymmetric. The asymmetry could be due to the accumulated error from the multi-layer patterning approach through repetition. We used the smallest $Z$ resolution, which means more layers and repetitions to build a structure. It is known that the lower $Z$ resolution results in a longer printing time and more artifacts and errors. The printed channel height and width were also smaller than the target dimensions. 
To determine the proper design height and width to create a microchannel with a width and height of $200 \mu \mathrm{m}$, we tried 5 different design heights $(240 \mu \mathrm{m}, 270 \mu \mathrm{m}, 300 \mu \mathrm{m}, 350 \mu \mathrm{m}, 380 \mu \mathrm{m})$ and 4 different widths $(120 \mu \mathrm{m}, 150 \mu \mathrm{m}, 180 \mu \mathrm{m}, 200 \mu \mathrm{m})$. To achieve a symmetrical channel profile, a correction structure is added on the right side of the design. The attempted correction structures are right-angle triangles with 3 different heights $(120 \mu \mathrm{m}, 150 \mu \mathrm{m}, 180 \mu \mathrm{m})$ with 2 different widths $(30 \mu \mathrm{m}, 60 \mu \mathrm{m})$. Figure 2 shows the definition of the correction structure height and width and its location with respect to the cross-sectional view of the printed structure. In order to test the influence of the printing temperature, we tried two different temperatures $\left(25^{\circ} \mathrm{C}\right.$ and $\left.40^{\circ} \mathrm{C}\right)$.

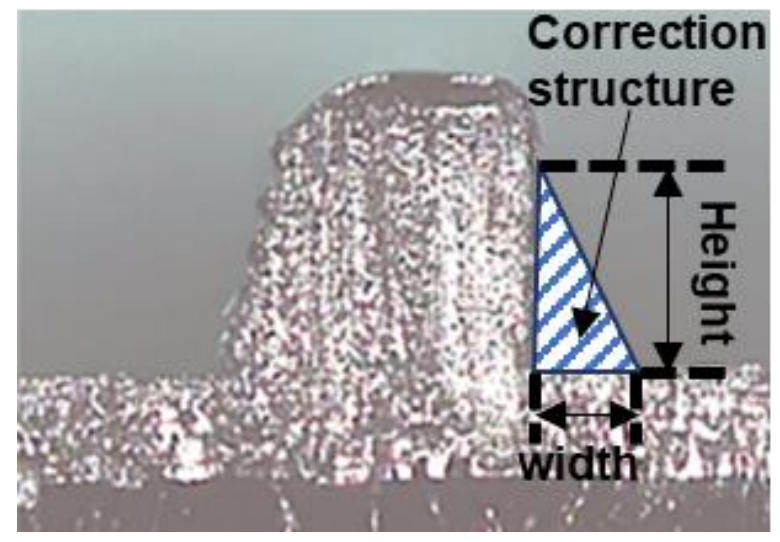

Figure 2 Definition and location of the correction structure.

Figure 3 shows the measured height and width of the 3D-printed microchannel at the printing temperature of $40{ }^{\circ} \mathrm{C}$ (blue dotted-dashed line) or $25{ }^{\circ} \mathrm{C}$ (green dotted line) with respect to the design parameters (red line). Results above the red line indicate the measured dimension is larger than the design parameter while the results below the red line mean the measured dimension is smaller than the design parameter. For both printing temperatures, 3D-printed channel height and width increase linearly as the design parameters increase. As shown in Figure 3(a), 3D-printed heights are $32 \pm 7.3 \%$ (at $40^{\circ} \mathrm{C}$ ) or $41 \pm 7.1 \%$ (at $25^{\circ} \mathrm{C}$ ) smaller than the design heights while the widths (Figure $3(\mathrm{~b})$ ) are $28 \pm 6.7 \%$ (at $40^{\circ} \mathrm{C}$ ) or $20 \pm 8.5 \%$ (at $25^{\circ} \mathrm{C}$ ) larger than the design widths.

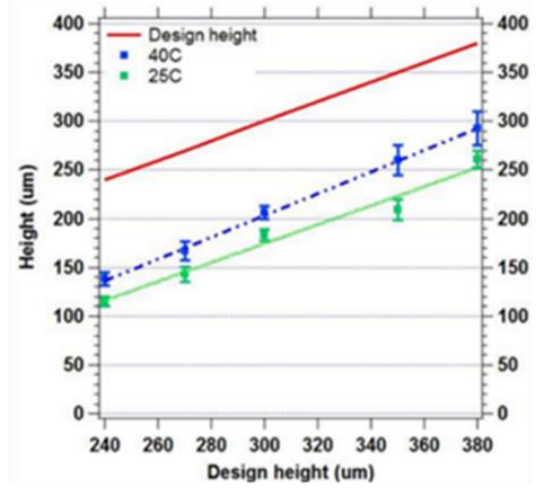

a

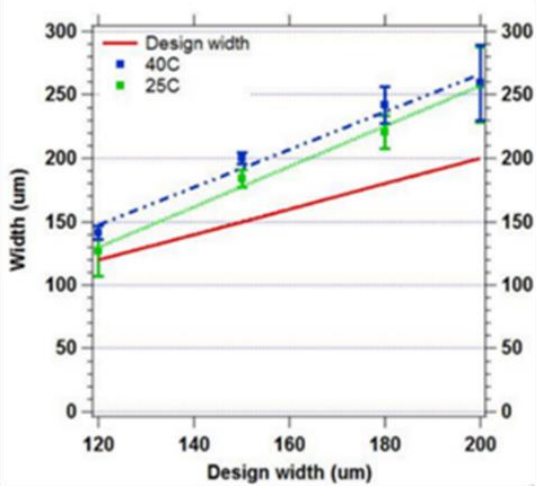

b

Figure 3 Comparison of design parameters and actual printed microchannel dimensions with $25^{\circ} \mathrm{C}$ and $40^{\circ} \mathrm{C}$ resin temperature. (a) Printed height VS design height. (b) Printed width VS design width. 
From the results, the optimum design parameters to be used to achieve the microchannel with the width and height of $200 \mu \mathrm{m}$ were determined to be $300 \mu \mathrm{m}$ for the height and $150 \mu \mathrm{m}$ for the width at $40{ }^{\circ} \mathrm{C}$. With these parameters, we found that a correction structure with a height of 180 $\mu \mathrm{m}$ and a width of $60 \mu \mathrm{m}$ makes the microchannel round and symmetric. The cross-sectional view of the 3D-printed microchannel using optimum parameters is shown in Figure 4. The 3D-printed microchannel has a symmetrical round shape with a height of $206 \pm 6.3 \mu \mathrm{m}$ and a width of $200 \pm 4.2$ $\mu \mathrm{m}$ which are close to the target dimension of $200 \mu \mathrm{m}$.

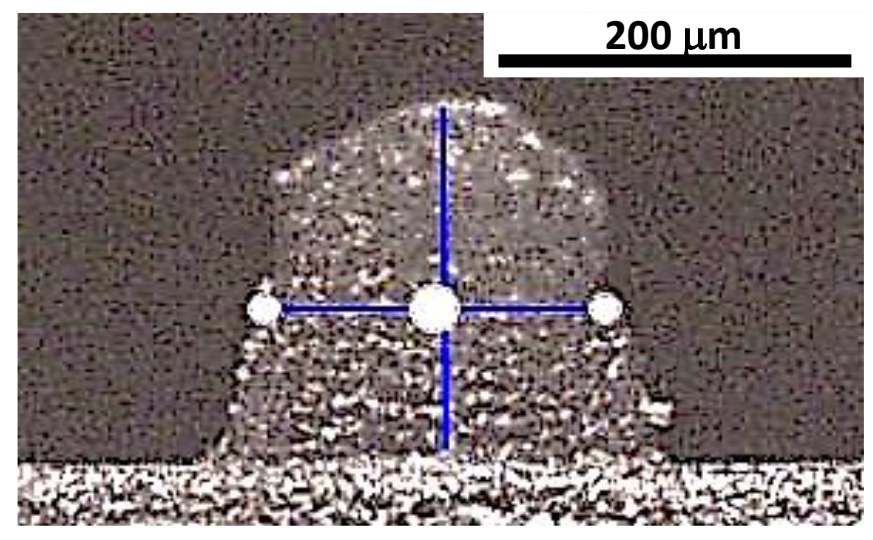

Figure 4 Cross-section view of the 3D-printed microchannel with the optimum design parameters and a correction structure.

\subsection{Surface Roughness of 3D Printed Mold Master}

We measured the surface roughness of 3D printed mold masters with the black resin using an Atomic Force Microscope (AFM), as shown in Figure 5. An area of $30 \mu \mathrm{m} \times 30 \mu \mathrm{m}$ was scanned at three different locations for each resin temperature and analyzed. With a resin temperature of $25^{\circ} \mathrm{C}$, the measured root-mean-square (RMS) roughness was $84.7 \pm 22 \mathrm{~nm}$ while the RMS roughness with $40{ }^{\circ} \mathrm{C}$ was slightly increased to $100.97 \pm 16 \mathrm{~nm}$. Overall, we were able to achieve a reasonably smooth surface.
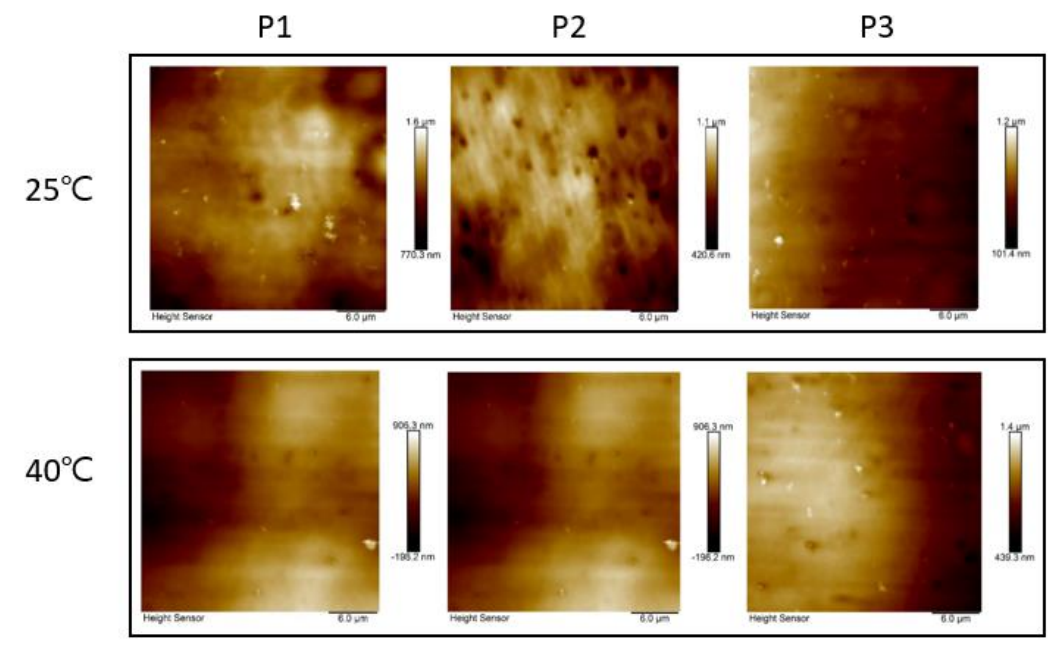

Figure 5 AFM images of the 3D printed mold masters with two different resin temperatures $\left(25^{\circ} \mathrm{C}\right.$ and $\left.40^{\circ} \mathrm{C}\right)$ at three areas. 


\subsection{Demonstration of Taper Structure Printing Capability of DLP 3D Printer}

As mentioned earlier, one of the unique benefits of 3D printing is the capability to create complex structures especially in the vertical direction. One of the challenging structures to fabricate with the conventional photolithography processes is a taper structure in the vertical direction. We conducted experiments to demonstrate the printing accuracy of B9 Creator for the taper structures. The test structures have a straight channel section and taper structures at the end with different angles in both horizontal and vertical directions as shown in Figure 6(a). The optimum design parameters and the correction structure determined from the previous experiments were used for the straight microchannel section so that the microchannel has a height and width of $200 \mu \mathrm{m}$ with a symmetric profile. Six different angles from $10^{\circ}$ to $60^{\circ}$ with an increment of $10^{\circ}$ were attempted as shown in Figure $6(b)$. The horizontal angle of the fabricated structure was measured from the top while the vertical angle was measured from the side.

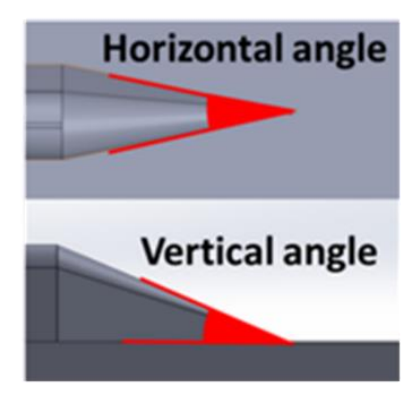

a

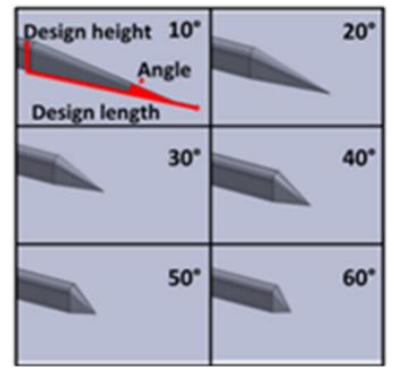

b

Figure 6 (a) Definition of the horizontal and vertical angles and (b) design of taper structures with 6 different angles.

The measurement results and microscope images of the taper test structures are shown in Figure 7. Figure 7(a) shows the measurement results of taper angles with respect to the design angle (red). The angle in the horizontal direction is slightly larger compared to the design (design angle $+1.8 \pm$ $1.7^{\circ}$ ) while the vertical angle is slightly smaller than the design (design angle $-2.14 \pm 0.9^{\circ}$ ).

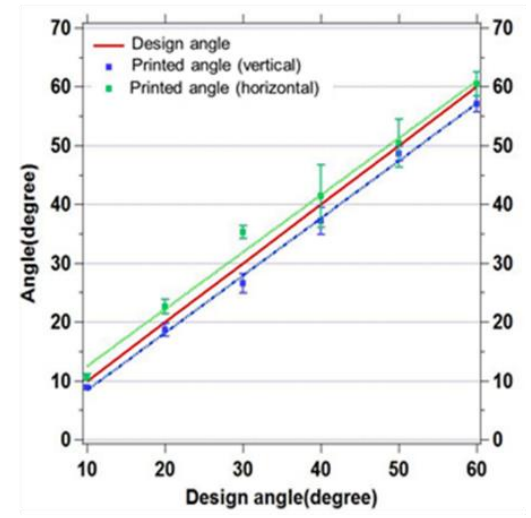

a

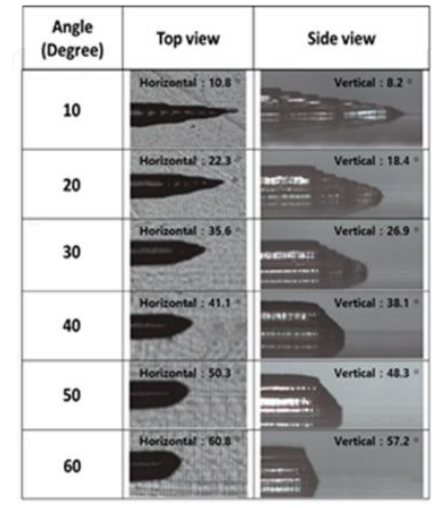

b

Figure 7 (a) Measurement results and (b) microscope images of printed taper structure compared to the design angle (printing temperature: $40^{\circ} \mathrm{C}$ ). 
Figure $7(b)$ shows the microscope images of the printed taper structures from the top and the side. As shown in the figure, the taper structures in the horizontal direction with an angle of $50^{\circ}$ and $60^{\circ}$ are slightly off-centered. By modifying the design accordingly, this error can be fixed. The vertical taper structures show staircase-like shapes due to the layer-by-layer printing method. If necessary, a polishing technique, such as an acetone bath, can be employed to smoothen the surface.

\subsection{Fabrication and Demonstration of a Microfluidic Device}

To demonstrate a 3D printing/PDMS integrated microfluidic device fabricated with B9 Creator, a simple device was designed and fabricated. Figure 8 shows the schematic drawing of the device. Input and output ports have a dimension of $2 \mathrm{~mm} \times 2 \mathrm{~mm}$ to allow a Tygon tubing (OD: 0.078") to be attached to the device. The input and output ports are tapered down with an angle of $55^{\circ}$ to microchannels. The input microchannel has a width and height of $200 \mu \mathrm{m}$. The output microchannel has a width of $250 \mu \mathrm{m}$ and a height of $200 \mu \mathrm{m}$. These two channels meet at the T-junction. At the end of the input microchannel after the T-junction, the microchannel is tapered down further with an angle of $27^{\circ}$. This channel is then connected to the output port through a $60 \mu \mathrm{m} \times 60 \mu \mathrm{m}$ microchannel. There are two normally open valves in the design to control the fluid flow in two different paths. Valve 1 is located on the input microchannel right after the T-junction while valve 2 is located on the output microchannel. By actuating valve 1 (V1 closed), the fluid from the input port will flow along the output channel at the T-junction. When valve 2 is actuated (V2 closed), the fluid from the input port flows through the T-junction, a tapered section with an angle of $27^{\circ}$, and then $60 \times 60$ microchannel reaching the output port. Valves are actuated through pneumatic channels and ports which are printed as part of the 3D-printed substrate.

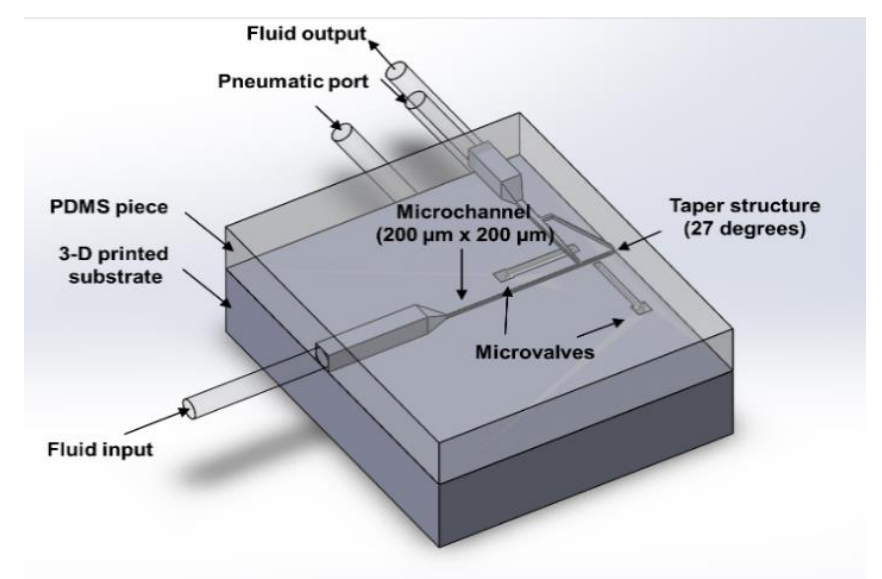

Figure 8 Schematic drawing of a microfluidic device.

The detailed fabrication processes are shown in Figure 9. The mold master for the PDMS piece was 3D printed using the design parameters determined from the previous experiments (Step 1). Then, the mold master was treated with TMCS. The SYLGARD 184 silicone elastomer mixed with the curing agent at a 1:10 (curing-agent: base) weight ratio was degassed in a chamber for 1 hour 30 min at $-100 \mathrm{kPa}$. The degassed PDMS was poured on the TMCS treated mold master (Step 2) and baked in an oven at $65^{\circ} \mathrm{C}$ for 2 hours. After baking, the PDMS piece was cut along the edge of the mold and gently peeled off from the mold master (Step 3). Figure 9 (b) shows the operation principle 
of a normally open microvalve used to control the fluid flow. For the operation of the microvalve, a thin PDMS membrane sits on top of a pneumatic pressure port. Normally, the valve is open, however, as pneumatic pressure is applied, the membrane is deformed and closes the channel. To fabricate the PDMS membrane, 1:10 ratio mixed PDMS was spin-coated at $3150 \mathrm{rpm}$ for $1 \mathrm{~min}$. on a TMCS treated 3-inch silicon substrate followed by a partial curing process at $55^{\circ} \mathrm{C}$ for $10 \mathrm{~min}$. on a hot plate. The PDMS piece from step 3 was gently placed on the partially cured PDMS membrane and was baked further at $100{ }^{\circ} \mathrm{C}$ for $10 \mathrm{~min}$. for bonding (Step 4) [31]. Then, the final PDMS piece was cut along the edge and gently peeled off from the Si substrate.

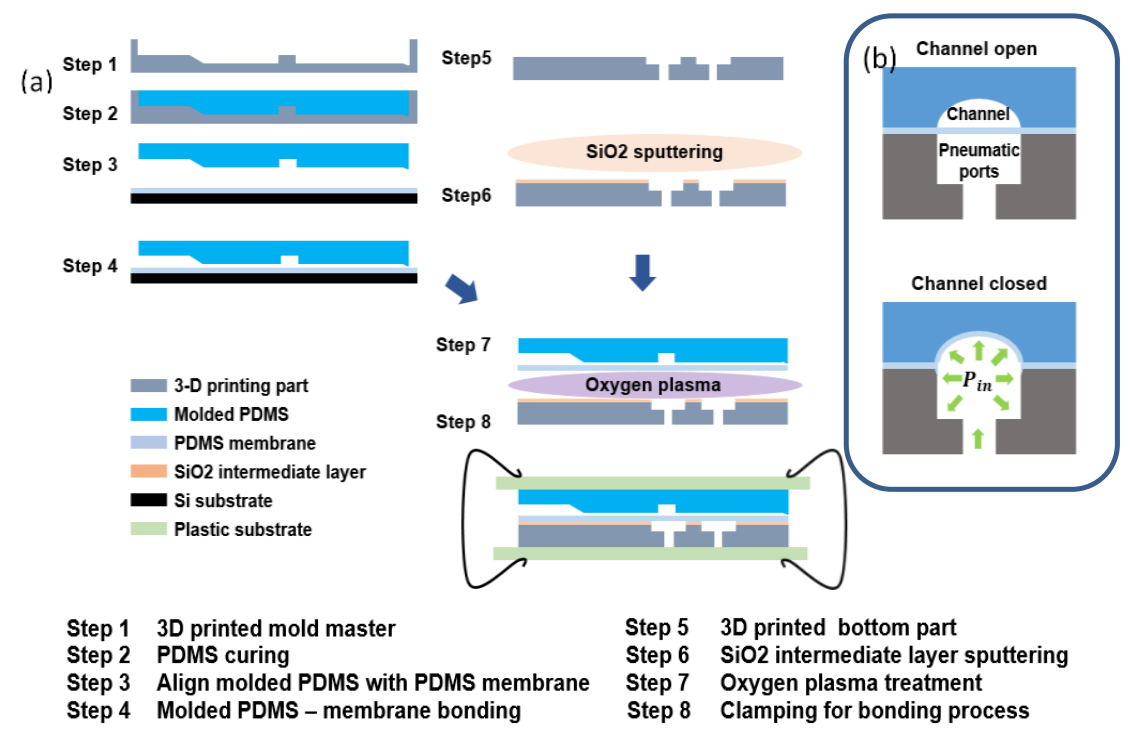

Figure 9 (a) Fabrication process of a microfluidic device. (b) Illustration of a normally open microfluidic valve and its operation.

To bond the top PDMS part with the bottom 3D-printed part, a thin layer of SiO2 $(77 \mathrm{~nm})$ was sputter-coated on the 3D-printed part (Step 6). Both PDMS and 3D- printed part surfaces to be bonded were exposed with oxygen plasma and clamped together for 1 hour at room temperature to achieve a strong and uniform bonding [29] (Steps 7 and 8). The fabricated device before and after attaching Tygon tubing (inner diameter (ID): 0.04") with epoxy glue is shown in Figures 10(a) and 10(b), respectively. The Tygon tubings for the pneumatic ports are then filled with DI water before the experiments.
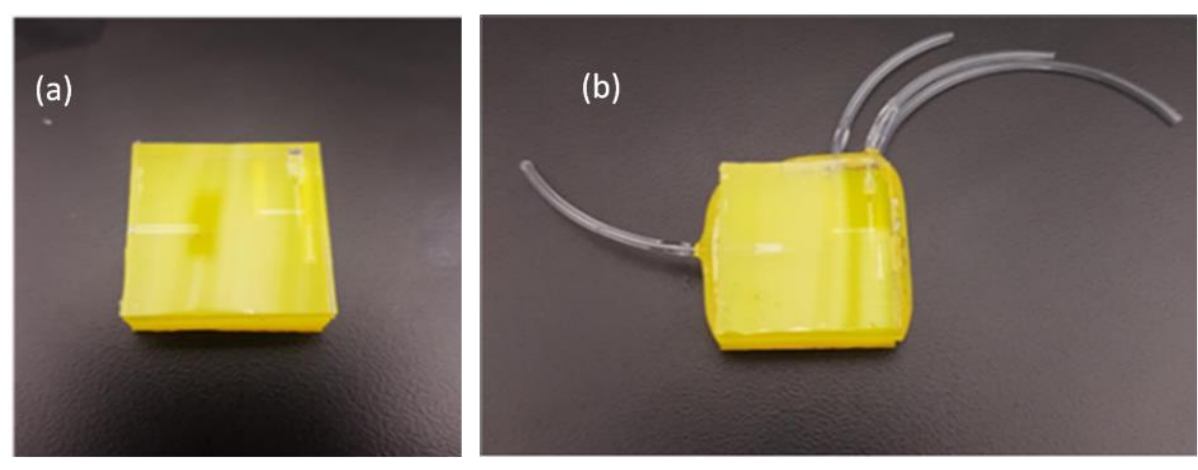

Figure 10 Fabricated microfluidic device (a) before and (b) after attaching tubings. 
Figure 11 shows a control system to test a fabricated microfluidic device. An infusion/withdrawal syringe pump (EW-74905-04, Cole-Parmer, USA) was used to introduce fluids. For the valve control, two solenoid valves (MHA1-M1LH-3/2G-0.6-PI, Wolf-Solution, USA) mounted in a solenoid valve manifold (CECC-D, Wolf-Solution, USA) were controlled by a controller (CECC-D, Wolf-Solution, USA).

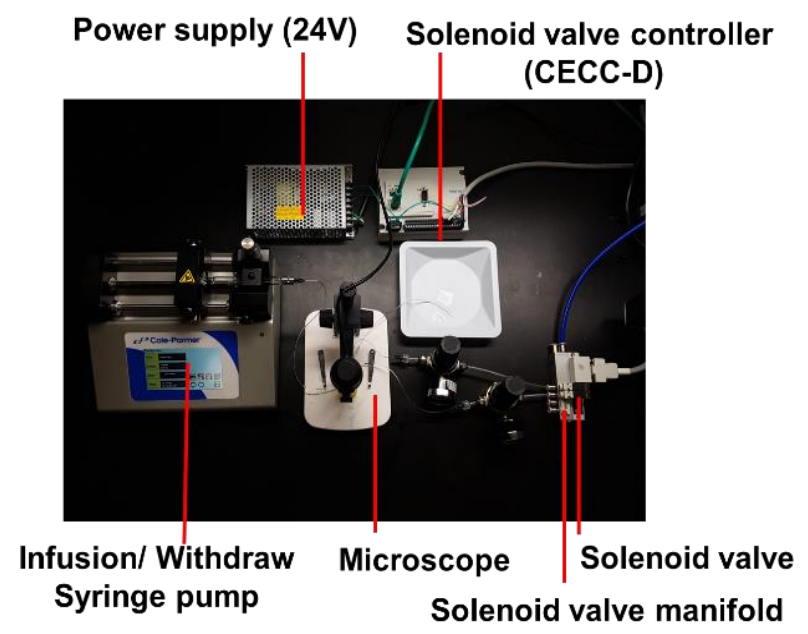

Figure 11 The control system.

Figure 12(a) shows the valve operation. We assigned V1 (Valve 1) and V2 (Valve 2) for two valves in the device. Without pressure applied to the pneumatic ports, the microchannel is open as shown in Figures 12(a),(i) (V1) and 12(a),(iii) (V2). Upon applied pressure to the ports, the PDMS membrane is expanded and closes the microchannel as seen in Figures 12(a),(ii) (V1) and 12(a),(iv) (V2). While V1 was open and V2 was closed, a blue dye was introduced into the device with a flow rate of 10 $\mu \mathrm{L} / \mathrm{min}$. as shown in the upper images of Figure 12(b). Then, a red dye was introduced into the channel while V1 was closed and V2 was open as shown in the lower images of Figure 12(b). The results clearly show this 3D printing/PDMS integrated microfluidic device works properly to direct the fluid flow with two valves.

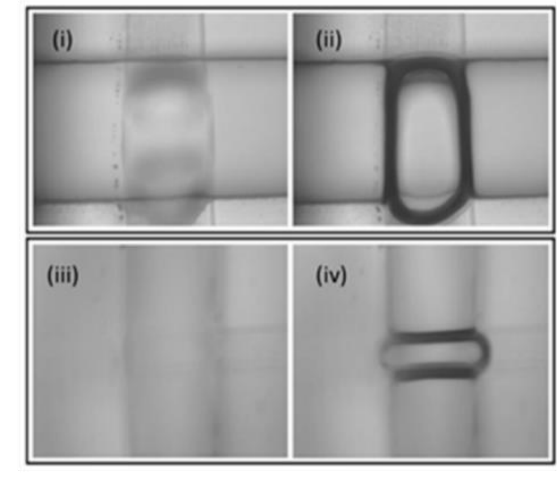

a

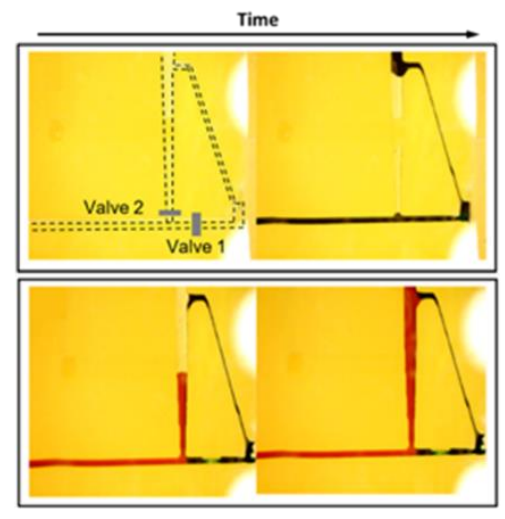

b

Figure 12 (a) The valve operation of a 3D-printing/PDMS integrated microfluidic device ((i): V1 open, (ii): V1 closed, (iii): V2 open, and (iv): V2 closed. (b) The fluid control demonstration of a 3D-printing/PDMS integrated microfluidic device (the upper images: V1 open and V2 closed, the lower images: V1 closed and V2 open). 


\section{Conclusions}

DLP 3D printer is an attractive tool to fabricate PDMS mold masters and microfluidic device substrates due to its fast-printing speed, high resolution, capability to create complex 3D structures and smooth printed surface. By integrating PDMS and 3D-printed substrate, various microfluidic devices can be fabricated while the fabrication process can be simplified. With the design height of $300 \mu \mathrm{m}$ and width of $150 \mu \mathrm{m}$ combined with an error correction structure on one side of design with a height of $180 \mu \mathrm{m}$ and a width of $60 \mu \mathrm{m}$, a symmetric and round shape microchannel with a height of $200 \mu \mathrm{m}$ and a width of $200 \mu \mathrm{m}$ was achieved. We successfully fabricated taper structures with various horizontal and vertical angles and showed the capability of the DLP 3D printer to fabricate such complex structures which can't be done with conventional photolithography processes. Finally, we successfully fabricated and demonstrated a microfluidic device integrating PDMS, molded with a 3D-printed mold master, and 3D-printed substrate. Using blue and red dye solutions, we showed that the microvalves function properly and the microfluidic device works as expected to control fluid flow.

\section{Author Contributions}

J.C.: design, fabrication, experimentation, investigation, analysis, and original draft preparation. S.K.: conceptualization, methodology, analysis, supervision, project management, and review and edit the manuscript.

\section{Competing Interests}

The authors have declared that no competing interests exist.

\section{References}

1. Vincent AG, Pascal RW, Beaton AD, Walk J, Hopkins JE, Woodward EM, et al. Nitrate drawdown during a shelf sea spring bloom revealed using a novel microfluidic in situ chemical sensor deployed within an autonomous underwater glider. Mar Chem. 2018; 205: 29-36.

2. Samuel R, Feng H, Jafek A, Despain D, Jenkins T, Gale B. Microfluidic-based sperm sorting \& analysis for treatment of male infertility. Transl Androl Urol. 2018; 7: S336-S347.

3. Szulwach KE, Chen P, Wang XH, Wang J, Weaver LS, Gonzales ML, et al. Single-cell genetic analysis using automated microfluidics to resolve somatic mosaicism. PLOS ONE. 2015; 10: e0135007.

4. Song J, Ryu H, Chung M, Kim Y, Blum Y, Lee SS, et al. Microfluidic platform for single cell analysis under dynamic spatial and temporal stimulation. Biosens Bioelectron. 2018; 104: 58-64.

5. Liu W, Song J, Du X, Zhou Y, Li Y, Li R, et al. AKR1B10 (Aldo-keto reductase family 1 B10) promotes brain metastasis of lung cancer cells in a multi-organ microfluidic chip model. Acta Biomater. 2019; 91: 195-208.

6. Liu J, Sternberg AR, Ghiasvand S, Berdichevsky Y. Epilepsy-on-a-chip system for antiepileptic drug discovery. IEEE Trans Biomed Eng. 2019; 66: 1231-1241.

7. Kaur G, Tomar M, Gupta V. Development of a microfluidic electrochemical biosensor: Prospect for point-of-care cholesterol monitoring. Sens Actuators B Chem. 2018; 261: 460-466. 
8. Rho D, Kim S. Label-free real-time detection of biotinylated bovine serum albumin using a lowcost optical cavity-based biosensor. Opt Express. 2018; 26: 18982-18989.

9. Zhou L, Zhuang G, Li G. A facile method for the fabrication of glass-PDMS-glass sandwich microfluidic devices by sacrificial molding. Sens Actuators B Chem. 2018; 261: 364-371.

10. Fan $W$, Qiao $M$, Jin $Y$, Zhou $H, G e ~ Y$, Jin $Q$, et al. High efficiency single-cell capture based on microfluidics for single cell analysis. J Micromech Microeng. 2019; 29: 035004.

11. Kaganovitch E, Steurer X, Dogan D, Probst C, Wiechert W, Kohlheyer D. Microbial single-cell analysis in picoliter-sized batch cultivation chambers. N Biotechnol. 2018; 47: 50-59.

12. Gao Y, Stybayeva G, Revzin A. Fabrication of composite microfluidic devices for local control of oxygen tension in cell cultures. Lab Chip. 2019; 19: 306-315.

13. Ren Y, Huang SH, Mosser S, Heuschkel MO, Bertsch A, Fraering PC, et al. A simple and reliable PDMS and SU-8 irreversible bonding method and its application on a microfluidic-MEA device for neuroscience research. Micromachines. 2015; 6: 1923-1934.

14. McCormick S, Tong Z, Ivask A, Morozesk M, Voelcker NH, Lombi E, et al. Optimization of binding B-lymphocytes in a microfluidic channel: Surface modification, stasis time and shear response. Biofabrication. 2017; 10: 014101.

15. Tang SY, Qiao R, Yan S, Yuan D, Zhao Q, Yun G, et al. Microfluidic mass production of stabilized and stealthy liquid metal nanoparticles. Small. 2018; 14: 1800118.

16. Shakoor A, Xie M, Luo T, Hou J, Shen Y, Mills JK, et al. Achieve automated organelle biopsy on small single cells using a cell surgery robotic system. IEEE Trans Biomed Eng. 2018; 66: 22102222.

17. Nakajima M, Ayamura Y, Takeuchi M, Hisamoto N, Pastuhov S, Hasegawa Y, et al. High-precision microinjection of microbeads into C. elegans trapped in a suction microchannel. Proceedings of the 2017 IEEE International Conference on Robotics and Automation (ICRA); 2017 May 29-June 3; 2017.

18. Lee YS. 3D-printed microfluidic control systems. Seattle: University of Washington; 2018.

19. Kitson PJ, Rosnes MH, Sans V, Dragone V, Cronin L. Configurable 3D-printed millifluidic and microfluidic 'lab on a chip' reactionware devices. Lab Chip. 2012; 12: 3267-3271.

20. Lee MP, Cooper GJ, Hinkley T, Gibson GM, Padgett MJ, Cronin L. Development of a 3D printer using scanning projection stereolithography. Sci Rep. 2015; 5. doi: 10.1038/srep09875.

21. Temiz Y, Lovchik RD, Kaigala GV, Delamarche E. Lab-on-a-chip devices: How to close and plug the lab? Microelectron Eng. 2015; 132: 156-175.

22. Amin R, Knowlton S, Hart A, Yenilmez B, Ghaderinezhad F, Katebifar S, et al. 3D-printed microfluidic devices. Biofabrication. 2016; 8: 022001.

23. Lee W, Kwon D, Choi W, Jung GY, Au AK, Folch A, et al. 3D-printed microfluidic device for the detection of pathogenic bacteria using size-based separation in helical channel with trapezoid cross-section. Sci Rep. 2015; 5: 7717.

24. Chan HN, Chen Y, Shu Y, Chen Y, Tian Q, Wu H. Direct, one-step molding of 3D-printed structures for convenient fabrication of truly 3D PDMS microfluidic chips. Microfluid Nanofluidics. 2015; 19: 9-18.

25. Rogers $\mathrm{Cl}$, Qaderi K, Woolley AT, Nordin GP. 3D printed microfluidic devices with integrated valves. Biomicrofluidics. 2015; 9: 016501. 
26. Gross BC, Anderson KB, Meisel JE, McNitt MI, Spence DM. Polymer coatings in 3D-printed fluidic device channels for improved cellular adherence prior to electrical lysis. Anal Chem. 2015; 87: 6335-6341.

27. Brennan MD, Rexius-Hall ML, Eddington DT. A 3D-printed oxygen control insert for a 24-well plate. PLOS ONE. 2015; 10: e0137631.

28. Tsuda S, Jaffery H, Doran D, Hezwani M, Robbins PJ, Yoshida M, et al. Customizable 3D printed 'plug and play' millifluidic devices for programmable fluidics. PLOS ONE. 2015; 10: e0141640.

29. Cheon J, Kim S. Intermediate layer-based bonding techniques for polydimethylsiloxane/digital light processing 3D-printed microfluidic devices. J Micromech Microeng. 2019; 29: 095005.

30. Dempsey D, McDonald S, Masato D, Barry C. Characterization of stereolithography printed soft tooling for micro injection molding. Micromachines. 2020; 11: 819.

31. Kim J, Surapaneni R, Gale BK. Rapid prototyping of microfluidic systems using a PDMS/ polymer tape composite. Lab Chip. 2009; 9: 1290-1293.

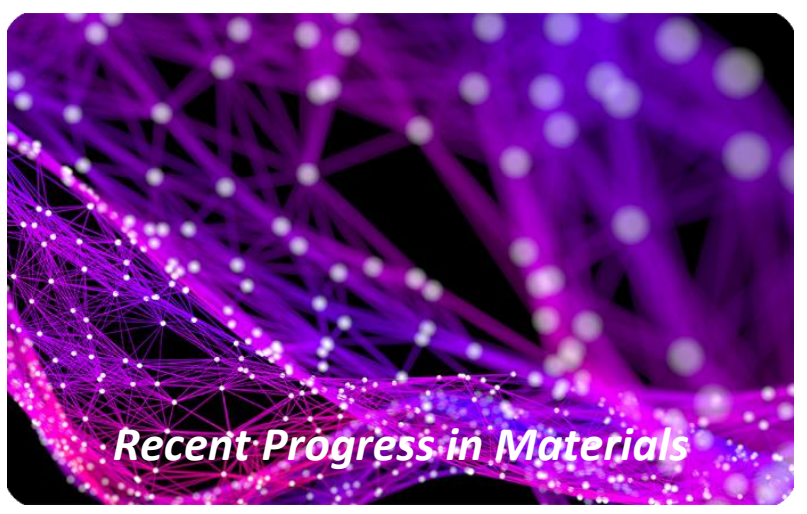

Enjoy Recent Progress in Materials by:

1. Submitting a manuscript

2. Joining in volunteer reviewer bank

3. Joining Editorial Board

4. Guest editing a special issue

For more details, please visit:

http://www.lidsen.com/journals/rpm 